\title{
GLOBAL EXISTENCE FOR 1D, COMPRESSIBLE, ISENTROPIC NAVIER-STOKES EQUATIONS WITH LARGE INITIAL DATA
}

\author{
DAVID HOFF
}

\begin{abstract}
We prove the global existence of weak solutions of the Cauchy problem for the Navier-Stokes equations of compressible, isentropic flow of a polytropic gas in one space dimension. The initial velocity and density are assumed to be in $L^{2}$ and $L^{2} \cap B V$ respectively, modulo additive constants. In particular, no smallness assumptions are made about the intial data. In addition, we prove a result concerning the asymptotic decay of discontinuities in the solution when the adiabatic constant exceeds $3 / 2$.
\end{abstract}

1. Introduction. In this paper we prove the existence of global weak solutions for the Navier-Stokes equations of compressible, isentropic flow

$$
\begin{aligned}
& v_{t}-u_{x}=0, \\
u_{t}+p(v)_{x}= & \left(\varepsilon u_{x}\right)_{x}, \quad x \in \mathbf{R}, t>0,
\end{aligned}
$$

with large, discontinuous initial data

$$
(v(x, 0), u(x, 0))=\left(v_{0}(x), u_{0}(x)\right) .
$$

Here $v, u$, and $p$ are the specific volume, velocity, and pressure in the fluid, and (1.1) and (1.2) are respectively the equations of conservation of mass and balance of forces. We assume throughout that

$$
p(v)=v^{-\gamma}, \quad \gamma>1,
$$

and

$$
\varepsilon(v)=b / v, \quad b>0 .
$$

Specifically, we prove the following theorem:

THEOREM 1.1. Assume that the initial data $v_{0}$ and $u_{0}$ satisfy $0<\underline{v}(0) \leq$ $v_{0}(x) \leq \bar{v}(0), v_{0}-v^{\prime} \in L^{2} \cap B V$ for some fixed $v^{\prime}>0$, and $u_{0} \in L^{2}$. Then the Cauchy problem (1.1)-(1.3) has a weak solution defined for all $t \geq 0$. The solution satisfies

$$
\begin{gathered}
0<\underline{v}(t) \leq v(x, t) \leq \bar{v}(t), \\
v(\cdot, t)-v^{\prime}, u(\cdot, t) \in L^{2}(\mathbf{R}),
\end{gathered}
$$

and

$$
\varepsilon(v)^{1 / 2} u_{x} \in L^{2}(\mathbf{R} \times[0, \infty]) .
$$

Received by the editors August 11, 1986.

1980 Mathematics Subject Classification (1985 Revision). Primary 76N15; Secondary 35K55.

This research was supported in part by the NSF under Grant No. MCS-8301141. 
The proof of this theorem is given in $\S 2$. In $\S 3$ we give a result concerning the qualitative behavior of the solution when the initial data contains a single jump discontinuity.

These results represent a continuation of our previous work [1] in which global existence for (1.1)-(1.3) was proved for initial data which was small in an appropriate sense. Our basic approach is, as before, to construct solutions as limits of approximate solutions obtained from a semidiscrete difference scheme. This enables us to bypass the need for a local existence theorem, as well as to avoid a priori smoothness assumptions. A few of the estimates used here are the same as in [1]. We shall therefore quote these estimates but omit their proofs.

The basic problem is to control the variable $v$ pointwise, so that the nonlinear terms $p(v)$ and $\varepsilon(v)$ continue to be bounded. In [1] this was achieved by establishing piecewise $H^{1}$ estimates for $v$ and estimates for the evolution in time of the jumps in $v$, then passing to a limit as the discretization tended to zero. The fact that these estimates could be made time-independent was a direct consequence of the smallness of the initial data. In this paper we instead give a discrete version of the argument of Kazhikov and Shelukhin, [5], modified to take account of the jump discontinuities and infinite spatial domain, to derive bounds for $v$ which do depend on time.

We now give a brief, heuristic discussion of the behavior of discontinuities in solutions of (1.1)-(1.2). Suppose that $(v, u)$ is such a solution, and that $v$ and $u$ are smooth except along a curve $\dot{x}=s$. The Rankine-Hugoniot relations applied to $(1.1)-(1.2)$ then imply that

$$
s[v]=-[u] \quad \text { and } \quad s[u]=\left[p-\varepsilon u_{x}\right] .
$$

(Here [.] denotes a jump across the discontinuity curve.) However, a result of Hoff-Smoller $[3]$ requires that $u$ must be smooth for $t$ positive, but that initial discontinuities in $v$ persist for all $t$. We therefore conclude that

$$
s=0 \quad \text { and } \quad[p(v)]=\left[\varepsilon(v) u_{x}\right] .
$$

That is, we expect that discontinuities in $u$ are smoothed out instantly, but that discontinuities in $v$ propagate along the lines $x=$ const. (which are particle paths in the Lagrangian equations (1.1)-(1.2)).

It is noteworthy that these lines of discontinuity do not in fact appear in the solutions of the conservation laws corresponding to (1.1)-(1.2), (i.e., equations (1.1)$(1.2)$ with $\varepsilon(v) \equiv 0)$. It is therefore of some interest to study the evolution in time of the jump in $p(v)$ (hence the jumps in $v$ and in $u_{x}$ ), as well as its dependence on the viscosity parameter $b$. We carry out such a discussion in $\S 3$, where we restrict attention to initial data with a single discontinuity. As a consequence of our analysis in $\S 2$, it is easy to see that, if there are time-independent bounds for the variable $v(x, t)$, then the jumps in $p(v), v$, and $u_{x}$ all tend to zero, exponentially as $t \rightarrow \infty$, and that this decay is more rapid the closer $b$ is to zero. (These conclusions therefore hold, by our previous results [1], for small initial data.) In $\S 3$ we show that, even for large initial data, if the adiabatic constant $\gamma$ is larger than $3 / 2$, then these same conclusions hold: $v(x, t)$ is bounded pointwise independent of time, and the jump discontinuities in $p(v), v$ and $u_{x}$ decay to zero exponentially as $t \rightarrow \infty$. 
We believe that it would be of great interest to give a comprehensive comparison of the solutions of the viscous equations (1.1)-(1.2) with the solutions of the corresponding conservation laws. To some extent, our results in $\S 3$ are a first step in this direction. We also call attention here to the result of Hoff and Liu [2], in which it is proved that, if $\left(v_{0}, u_{0}\right)$ represents Riemann initial data for which the solution of the conservation laws corresponding to (1.1)-(1.2) consists of a single shock wave, then the solution of the Navier-Stokes equations (1.1)-(1.2) with the same initial data exists for all $t>0$ and tends to that shock wave as the viscosity parameter $b$ tends to zero. We also point out that global existence of solutions of (1.1)-(1.2) with general Riemann initial data is not yet proved; such data is excluded in the present paper by the requirement that $v_{0}-v^{\prime}$ and $u_{0}$ be in $L^{2}$. However, [2] does contain a proof of local existence in time for initial data having different limits at $+\infty$ and $-\infty$.

Existence theorems for the equations of compressible fluid flow also appear in $[\mathbf{3}, \mathbf{4}, \mathbf{5}$, and $\mathbf{6}]$. The reader may consult [1] for brief descriptions and comparisons of these papers.

(NOTE ADDED IN PROOF. It has come to our attention that an existence result similar to ours is obtained by D. Serre, [7]; he considers more general pressure functions, but requires that the initial density be in $L^{1}(\mathbf{R})$.)

2. Proof of Theorem 1.1. In this section we prove Theorem 1.1 by applying the semidiscrete difference scheme introduced in [1]. This scheme is described as follows. Let $h>0$ be an increment in $x$ and define $x_{k}=k h$. Approximations to $u\left(x_{k}, t\right)$ and $v\left(x_{k+1 / 2}, t\right)$ will be denoted by $u_{k}(t)$ and $v_{k+1 / 2}(t)$, and quantities such as $\varepsilon\left(v_{k+1 / 2}(t)\right)$ will be abbreviated by $\varepsilon_{k+1 / 2}(t)$, etc. We define the spatial difference operator $\delta$ by

$$
\delta w_{k}=\frac{w_{k+1 / 2}-w_{k-1 / 2}}{h} \quad \text { and } \quad \delta w_{k+1 / 2}=\frac{w_{k+1}-w_{k}}{h} .
$$

We assume that initial values $\left(v_{k+1 / 2}(0), u_{k}(0)\right)$ have been given for integral $k$, and that there are distinguished nodes $x_{k_{1}}<\cdots<x_{k_{m}}$ which may be thought of as the points of discontinuity of $v_{0}(x)$. Approximate solutions $u_{k}(t)$ and $v_{k+1 / 2}(t)$ are then computed form the odes

$$
\dot{v}_{k+1 / 2}=\delta u_{k+1 / 2}, \quad \text { all } k
$$

and

$$
\dot{u}_{k}=\delta(-p+\varepsilon \delta u)_{k}, \quad k \neq k_{i} .
$$

Observe that neither of the equations (1.1)-(1.2) is imposed along the discontinuity lines $x=x_{k_{i}}$. Instead, we require that

$$
[p]_{k_{i}}=[\varepsilon \delta u]_{k_{i}}
$$

that is,

$$
p_{k_{i}+1 / 2}-p_{k_{i}-1 / 2}=(\varepsilon \delta u)_{k_{i}+1 / 2}-(\varepsilon \delta u)_{k_{i}-1 / 2},
$$

which is the obvious discretization of the jump condition (1.9).

Solving (2.3) for $u_{k_{i}}$, we obtain

$$
u_{k_{i}}=\frac{\varepsilon_{k_{i}+1 / 2} u_{k_{i}+1}+\varepsilon_{k_{i}-1 / 2} u_{k_{i}-1}-h[p]_{k_{i}}}{\varepsilon_{k_{i}+1 / 2}+\varepsilon_{k_{i}-1 / 2}} .
$$


Thus as long as $v_{k+1 / 2}(t) \geq \underline{v}>0$ for all $k, u_{k_{i}}$ is a smooth function of the variables $u_{k}, k \neq k_{i}$, and $v_{k+1 / 2}$. Equations (2.1)-(2.3) then represent an ode in the space $l^{\infty}$, for which a unique solution therefore exists locally in time.

Define the functions $E(v)$ and $\psi(v)$ by

$$
E(v)=b \log v,
$$

so that $E^{\prime}(v)=\varepsilon(v)$, and

$$
\psi(v)=\int_{v^{\prime}}^{v}\left[p\left(v^{\prime}\right)-p(s)\right] d s=p\left(v^{\prime}\right)\left(v-v^{\prime}\right)+\frac{v^{1-\gamma}-\left(v^{\prime}\right)^{1-\gamma}}{\gamma-1},
$$

where $v^{\prime}>0$ is fixed. We then have the following global existence result for the scheme (2.1)-(2.3).

THEOREM 2.1. Assume that the initial data $\left(v_{j}(0), u_{k}(0)\right)$ for the scheme (2.1)(2.3) satisfies

$$
\sum_{k} u_{k}(0)^{2} h+\sum_{j} \psi\left(v_{j}(0)\right) h+\sum_{i}\left|[E]_{k_{i}}(0)\right| \leq K_{0}<\infty
$$

and

$$
0<1 / K_{0} \leq v_{j}(0) \leq K_{0} \quad \forall j .
$$

Then there is a solution $\left(v_{j}(t), u_{k}(t)\right)$ of $(2.1)-(2.3)$, defined for all $t>0$, satisfying

$$
\begin{gathered}
0<1 / C(T) \leq v_{j}(t) \leq C(T), \quad 0 \leq t \leq T ; \\
\sum_{k \neq k_{i}} u_{k}(t)^{2} h+\sum_{j} \psi_{j}(t) h+\int_{0}^{t} \sum_{j} \varepsilon_{j}(s) \delta u_{j}(s)^{2} h d s \leq C ; \\
\sum_{k \neq k_{i}} \delta v_{k}(t)^{2} h \leq C(T), \quad 0 \leq t \leq T ;
\end{gathered}
$$

and

$$
\left|[E]_{k_{i}}(t)\right| \leq\left|[E]_{k_{i}}(0)\right| \leq C .
$$

In the above statement, and throughout this section, $C$ will denote a generic positive constant which is independent of $t$ and of $h$, but which may depend on $K_{0}$. $C(t)$ is the same as $C$, excpet that $C(t)$ may depend on $t$. Also, without loss of generality, we may take $K_{0} \geq 1 \geq h$ throughout.

PROOF OF THEOREM 1.1. Theorem 1.1 now follows from Theorem 2.1 by converting the intitial data $\left(v_{0}(x), u_{0}(x)\right)$ to mesh functions $\left(v_{j}(0), u_{k}(0)\right)$, solving the scheme (2.1)-(2.3) with these mesh functions as data, and then passing to the limit as $h \rightarrow 0$, making use of the uniform estimates $(2.7)-(2.10)$. The details are nearly identical to those in the development in $\S 3$ of [1] , and so will be omitted. Q.E.D.

The proof of Theorem 2.1 will be given in a sequence of lemmas. We being with the following energy estimates, which are proved in Lemmas 2.2 and 2.3 of [1]:

LEMMA 2.1. Assume that the equations (2.1)-(2.3) have a solution defined up to time $T$ with $v_{j}(t)>0$ for $0 \leq t \leq T$. Then for $0 \leq t \leq T$,

$$
\sum_{k \neq k_{i}} u_{k}(t)^{2} h+\sum_{j} \psi_{j}(t) h+\int_{0}^{t} \sum_{j} \varepsilon_{j}(s) \delta u_{j}(s)^{2} h d s \leq C
$$


and

$$
\frac{1}{4} \sum_{k \neq k_{i}} \delta E_{k}(t)^{2} h \leq C+\sum_{i} \int_{0}^{t} u_{k_{i}}(s)[\dot{E}]_{k_{i}}(s) d s .
$$

In addition, the jump in $E(v)$ at $x_{k_{i}}$ satisfies

$$
[E]_{k_{i}}(t)=[E]_{k_{i}}(0) \exp \left(\int_{0}^{t} \alpha_{i}(s) d s\right)
$$

where

$$
\alpha_{i}(s)=[p]_{k_{i}}(s) /[E]_{k_{i}}(s) .
$$

Our immediate goal is to obtain pointwise bounds for $v_{j}(t)$ which are independent of $h$. Only (2.11) of Lemma 2.1 will be required in this argument. We begin with the following technical lemma.

LEMMA 2.2. Assume that the hypotheses of Lemma 2.1 are in force and fix $j$. Then for each $t \geq 0$ there is an integer $j^{\prime}=j^{\prime}(j, t)$, depending measurably on $t$, such that

$$
\begin{gathered}
\sum_{l=j+1}^{j^{\prime}(j, t)} v_{l}(t) h \geq 1, \\
v_{j^{\prime}(j, t)} \geq 1 / C>0,
\end{gathered}
$$

and

$$
\left|x_{j^{\prime}}-x_{j}\right| \leq C \text {. }
$$

PROOF. Fix $t_{0}>0$ and choose first $j^{\prime \prime}>j$ such that

$$
\sum_{l=j+1}^{j^{\prime \prime}-1} v_{l}\left(t_{0}\right) h \leq 1<\sum_{l=j+1}^{j^{\prime \prime}} v_{l}\left(t_{0}\right) h .
$$

(This is possible because $v_{l} \rightarrow v^{\prime}>0$ as $j \rightarrow \infty$.) Then choose $j^{\prime} \geq j^{\prime \prime}$ so that $v_{j^{\prime}}\left(t_{0}\right) \geq \min \left(1 / 2, v^{\prime} / 2\right)$. We need an upper bound for $\sum_{l=j+1}^{j^{\prime}} v_{l}\left(t_{0}\right) h$. First,

$$
\sum_{l=j^{\prime \prime}+1}^{j^{\prime}-1} v_{l}\left(t_{0}\right) h \leq \frac{1}{2}\left|x_{j^{\prime}-1}-x_{j^{\prime \prime}}\right| \leq \frac{1}{2}\left|x_{j^{\prime}}-x_{j}\right| .
$$

Next, let $l=j^{\prime}$ or $j^{\prime \prime}$. If $v_{l} \leq v^{\prime}+1$, then $v_{l} h \leq c$. Otherwise, if $v_{l}>v^{\prime}+1$, then by (2.6) and Lemma 2.1,

$$
v_{l} h \leq C \psi\left(v_{l}\right) h \leq C
$$

We therefore have that

$$
1<\sum_{l=j+1}^{j^{\prime}} v_{l}\left(t_{0}\right) h \leq C+\frac{1}{2}\left|x_{j^{\prime}}-x_{j}\right| .
$$

(2.18) together with the condition

$$
v_{j^{\prime}}\left(t_{0}\right) \geq \min \left[1 / 2, v^{\prime} / 2\right] \geq 1 / c
$$

enable us to define $j^{\prime}$ as a measurable function of $t$. 
To estimate $\left|x_{j^{\prime}}-x_{j}\right|$, we observe that $p=\gamma /(\gamma-1)$ and $q=\gamma$ are conjugate indices. Using (2.18) we then have that

$$
\left|x_{j^{\prime}}-x_{j}\right|=\sum_{l=j+1}^{j^{\prime}} v_{l}^{(\gamma-1) / \gamma} v_{l}^{(1-\gamma) / \gamma} h \leq\left(\sum v_{l} h\right)^{(\gamma-1) / \gamma}\left(\sum v_{l}^{1-\gamma} h\right)^{1 / \gamma}
$$

However, from (2.6),

$$
\psi(v) \sim v^{1-\gamma} \quad \text { as } v \rightarrow 0
$$

so that

$$
v^{1-\gamma} \leq C \psi(v)+1, \quad v>0
$$

Appealing to Lemma 2.1 again, we then have that

$$
\left|x_{j^{\prime}}-x_{j}\right| \leq\left[C+\frac{1}{2}\left|x_{j^{\prime}}-x_{j}\right|\right]^{(\gamma-1) / \gamma}\left[C+\left|x_{j^{\prime}}-x_{j}\right|\right]^{1 / \gamma} .
$$

This proves (2.17).

The next lemma is the discrete version of an argument given in [5], modified to take account of the jumps in $v$.

LEMMA 2.3. Assume that the hypotheses of Lemma 2.1 are in force. Fix $j$ and $t \in[0, T]$, and let $j^{\prime}=j^{\prime}(j, t)$ as in Lemma 2.2. Then for $l<j^{\prime}$,

$$
v_{l}(t) A(t)=\frac{v_{l}(0)+I_{l}(t)}{v_{j^{\prime}}(0) B_{l}(t)}
$$

where

$$
\begin{aligned}
A(t) & =\frac{\exp \left[b^{-1} \int_{0}^{t} p_{j^{\prime}}(s) d s\right]}{v_{j^{\prime}}(t)} \\
B_{l}(t) & =\exp \left[b^{-1} \sum_{\substack{k=l+1 / 2 \\
k \neq k_{i}}}^{j^{\prime}-1 / 2}\left(u_{k}(t)-u_{k}(0)\right) h\right]
\end{aligned}
$$

and

$$
I_{l}(t)=b^{-1} \int_{0}^{t} v_{j^{\prime}(j, s)}(0) A(s) B_{l}(s) p_{l}(s) v_{l}(s) d s
$$

PROOF. We have from (2.1), (2.2), and (2.5) that, for $k \neq k_{i}$,

$$
\delta \dot{E}_{k}=\delta(\varepsilon \delta u)_{k}=\dot{u}_{k}+\delta p_{k}
$$

Consequently,

$$
\left.\delta E_{k}(\cdot)\right|_{0} ^{s}=\left.u_{k}(\cdot)\right|_{0} ^{s}+\int_{0}^{s} \delta p_{k}(\tau) d \tau
$$


and for $l<j^{\prime}(j, s)$,

$$
\begin{aligned}
{\left.\left[E_{j^{\prime}(j, s)}(\cdot)-E_{l}(\cdot)\right]\right|_{0} ^{s}=} & \left.\sum_{\substack{l<k<j^{\prime}(j, s) \\
k \neq k_{i}}} \delta E_{k}\right|_{0} ^{s} h+\left.\sum_{l<k_{i}<j^{\prime}(j, s)}[E]_{k_{i}}\right|_{0} ^{s} \\
= & \left.\sum_{\substack{l<k<j^{\prime}(j, s) \\
k \neq k_{i}}} u_{k}(\cdot)\right|_{0} ^{s} h \\
& +\int_{0}^{s}\left[p_{j^{\prime}(j, s)}(\tau)-\sum_{l<k_{i}<j^{\prime}(j, s)}[p]_{k_{i}}(\tau)-p_{l}(\tau)\right] d \tau .
\end{aligned}
$$

But by (2.5), (2.1), and (2.3),

$$
[\dot{E}]_{k_{i}}=[\varepsilon \delta u]_{k_{i}}=[p]_{k_{i}}
$$

so that the jump terms above exactly cancel. Rearranging, we thus obtain

$$
\begin{aligned}
-E_{l}(s)+\int_{0}^{s} p_{l}(\tau) d \tau= & -E_{j^{\prime}(j, s)}+\int_{0}^{s} p_{j^{\prime}(j, s)}(\tau) d \tau \\
& +E_{j^{\prime}(j, s)}(0)-E_{l}(0)+b \log B_{l}(s) .
\end{aligned}
$$

Dividing by $b$ and taking exponentials, we then get

$$
\frac{\exp \left[b^{-1} \int_{0}^{s} p_{l}(\tau) d \tau\right]}{v_{l}(s)}=\frac{v_{j^{\prime}(j, s)}(0)}{v_{l}} A(s) B_{l}(s)
$$

and multiplying by $p_{l}(s) v_{l}(s)$,

$$
\frac{d}{d s} \exp \left[b^{-1} \int_{0}^{s} p_{l}(\tau) d \tau\right]=\frac{v_{j^{\prime}(j, s)}(0)}{b v_{l}(0)} A(s) B_{l}(s) p_{l}(s) v_{l}(s)
$$

Thus

$$
\exp \left[b^{-1} \int_{0}^{t} p_{l}(s) d s\right]=1+\frac{I_{l}(t)}{v_{l}(0)}
$$

Substituting this into (2.20) and rearranging, we then obtain (2.19).

Pointwise bounds for $v_{j}(t)$ will follow easily from (2.19) once we have estimated the quantities $A(t)$ and $B_{l}(t)$ appearing in Lemma 2.3 .

LEMMA 2.4. Assume that the hypotheses of Lemma 2.1 are in force. Then for $j \leq l \leq j^{\prime}(j, t)$,

$$
A(t) \leq C e^{C t}
$$

and

$$
0<1 / C \leq B_{l}(t) \leq C .
$$

PROOF. From Lemmas 2.1 and 2.2 we have that

$$
\left|\sum_{\substack{k=l+1 / 2 \\ k \neq k_{i}}}^{j^{\prime}-1 / 2}\left[u_{k}(t)-u_{k}(0)\right] h\right| \leq C \sqrt{x_{j^{\prime}}-x_{j}} \leq C .
$$


This proves (2.22). The proof of (2.21) is slightly more involved. If we sum over $l$ in (2.19) and use (2.15), we obtain

$$
\begin{aligned}
A(t) & <\sum_{l=j+1}^{j^{\prime}(j, t)} v_{l}(t) A(t) h \\
& \leq C \sum_{l=j+1}^{j^{\prime}(j, t)}\left[v_{l}(0)+I_{l}(t)\right] h .
\end{aligned}
$$

However,

$$
v \leq C[\psi(v)+1], \quad v>0
$$

by $(2.6)$, so that

$$
\sum_{l=j+1}^{j^{\prime}} v_{l}(0) h \leq C\left[1+\left|x_{j^{\prime}}-x_{j}\right|\right] \leq C
$$

by (2.8) and (2.16). Also,

$$
I_{l}(t) \leq C \int_{0}^{t} A(s) p_{l}(s) v_{l}(s) d s
$$

so that $(2.23)$ becomes

$$
A(t) \leq C+C \int_{0}^{t} A(s)\left[\sum_{l=j+1}^{j^{\prime}(j, t)} p_{l}(s) v_{l}(s) h\right] d s .
$$

If we knew that

$$
\sum_{l=-\infty}^{\infty} p_{l}(s) v_{l}(s) h \leq C
$$

then Gronwall's inequality applied to (2.24) would prove (2.21).

To prove (2.25) we first note that, from (2.1),

$$
\frac{d}{d t} \frac{u_{k}^{2}}{2}+u_{k} \delta(p-\varepsilon \delta u)_{k}=0, \quad k \neq k_{i}
$$

Summing by parts, we therefore obtain

$$
\frac{d}{d t} \frac{1}{2} \sum_{k \neq k_{i}} u_{k}^{2} h+\sum_{j} \varepsilon_{j} \delta u_{j}^{2} h-\sum_{j} p_{j} \delta u_{j} h=0,
$$

where we used the jump condition (2.3). Thus rearranging,

$$
\left|\int_{0}^{t} \sum_{j} p_{j}(s) \delta u_{j}(s) h d s\right| \leq C
$$


by Lemma 2.1. Returning to (2.25), we then have that, since $p^{\prime}(v) v=C p(v)$, and $\dot{v}_{l}=\delta u_{l}$

$$
\begin{aligned}
\left|\sum_{l} p_{l}(t) v_{l}(t) h\right| & \leq C+\left(\int_{0}^{t} \sum_{l}\left[p^{\prime}\left(v_{l}\right) v_{l}+p\left(v_{l}\right)\right] \dot{v}_{l} h d s\right) \\
& =C+C\left|\int_{0}^{t} \sum_{l} p_{l}(s) \delta u_{l}(s) h d s\right| \\
& \leq C
\end{aligned}
$$

as required.

PROOF OF THEOREM 2.1. From (2.19), (2.21) and (2.22), we have that $v_{l}(t) \geq C e^{-C t}$. Consequently $p_{l}(s) \leq C e^{C s}$, so that the integrand in the definition of $I_{l}(t)$ in Lemma 2.3 is bounded by $A(s) v_{l}(s) e^{C s}$. (2.19) then implies that

$$
v_{l}(t) A(t) \leq C+C \int_{0}^{t} v_{l}(s) A(s) e^{C s} d s .
$$

(2.7) then follows by Gronwall's inequality and (2.16). Since the nonlinear functions appearing in (2.1)-(2.3) are smooth and Lipschitz for $v \in[a, b] \subseteq(0, \infty)$, the bounds (2.7) imply that the odes (2.1) $-(2.3)$ are solvable globally in time. (2.8) is then a restatement of $(2.11) ;(2.10)$ follows from $(2.13)$ and the fact that $\alpha_{i}(s)$ is negative; and (2.9) will follow from (2.12) and (2.7) once we bound the term $\Sigma_{i} \int_{0}^{t} u_{k_{i}}(s)[\dot{E}]_{k_{i}}(s) d s$ appearing in (2.12).

To do this we first observe that, from (2.4),

$$
u_{k_{i}}=\tilde{u}_{k_{i}}+O(h)[p]_{k_{i}}
$$

where $\tilde{u}_{k_{i}}$ is a convex combination of $u_{k_{i}+1}$ and $u_{k_{i}-1}$. The pointwise bound (2.7) then implies that

$$
\sum_{k} u_{k}^{2} h=C(T)\left(\sum_{k \neq k_{i}} u_{k}^{2} h+h^{3}\right) \leq C(T)
$$

by Lemma 2.1 . Thus

$$
u_{k_{i}}^{2} \leq C\left[\sum_{k} u_{k}^{2} h+\sum_{j} \delta u_{j}^{2} h\right] \leq C(T)\left[1+\sum_{j} \delta u_{j}^{2} h\right] .
$$

We therefore have from (2.11) and 2.10) that

$$
\begin{aligned}
\left|\sum_{i} \int_{0}^{t} u_{k_{i}}(s)[\dot{E}]_{k_{i}}(s) d s\right| & \leq C(T) \sum_{i}\left|[E]_{k_{i}}(0)\right| \max _{i}\left(\int_{0}^{t} u_{k_{i}}(s)^{2} d s\right)^{1 / 2} \\
& \leq C(T)\left(\int_{0}^{t}\left[1+\delta u_{j}(s)^{2} h\right] d s\right)^{1 / 2} \leq C(T)
\end{aligned}
$$

as required. 
3. Decay of the discontinuities in density. In this section we focus attention on the discontinuities in the solution (1.1)-(1.2). These jumps evidently persist for all time along the lines $x=$ const. It is noteworthy that these discontinuities fail to appear in the solutions of the conservation laws corresponding to (1.1)-(1.2) (i.e., the equations (1.1)-(1.2) with $\varepsilon(v) \equiv 0)$.

We recall from Lemma 2.1 that, for the approximate solution constructed in $\S 2$,

$$
[E]_{k_{i}}(t)=[E]_{k_{i}}(0) \exp \left[\int_{0}^{t} \alpha_{i}(s) d s\right]
$$

where $E(v)=b \log v$ (so that $E^{\prime}=\varepsilon$ ) and

$$
\alpha_{i}(s)=[p]_{k_{i}}(s) /[E]_{k_{i}}(s)=\frac{d p}{d E}(\tilde{E})=-\frac{\gamma}{b} \tilde{v}^{-\gamma}<0 .
$$

Consequently, if there were time-independent bounds for $v$,

$$
0<\underline{v} \leq v(x, t) \leq \bar{v}, \quad t \geq 0
$$

then (3.1) and (3.2) would imply that

$$
\left|[E]_{k_{i}}(t)\right| \leq\left|[E]_{k_{i}}(0)\right| \exp \left(-(\gamma / b) \underline{v}^{-\gamma} t\right) .
$$

Thus the jump in $E(v)$, hence the jumps in $v, p(v)$, and $u_{x}$ would decay to zero exponentially in $t$. Moreover, this rate of decay would be faster the closer $b$ is to 0 (i.e., the smaller the viscosity term in the momentum equation). We reamrk that, when the initial data $\left(v_{0}, u_{0}\right)$ is small (in an appropriate sense), the results of [1] apply to show that there are in fact such bounds (3.3), so that the jumps in $v$ do indeed decay to 0 .

We therefore consider intitial data as in Theorem 1.1, but in which $v_{0}$ contains just a single discontinuity at $x=0$, not necessarily small, and with $v_{0}-v^{\prime}$ in $H^{1}(x<0) \cap H^{1}(x>0)$. We ask whether there are indeed time-independent pointwise bounds for $v$, and whether the jump in $v(\cdot, t)$ does in fact decay to zero. The following theorem gives an affirmative answer at least for the case $\gamma>3 / 2$.

THEOREM 3.1. Assume that the initial data $\left(v_{0}, u_{0}\right)$ for (1.1)-(1.2) is as described above and that $p(v)=v^{-\gamma}$ with $\gamma>3 / 2$. Then there is a constant $C$, independent of time, such that the solution constructed in $\S 2$ satisfies

$$
0<1 / C \leq v(x, t) \leq C
$$

for all $x$ and $t$. In addition, $v(\cdot, t)-v^{\prime}$ is in $H^{1}(x<0) \cap H^{1}(x>0)$, and the jump $v(0+, t)-v(0-, t)$ decays to zero exponentially as $t \rightarrow \infty$.

ProOF. We introduce the function $F(v)$ defined by

$$
F(v)=\int_{v^{\prime}}^{v} \psi(s)^{1 / 2} \varepsilon(s) d s
$$

where $\psi$ is as in (2.6). An easy computation based on the definition of $\psi$ then shows that

$$
\begin{aligned}
v^{(1-\gamma) / 2} & \leq C F(v), & & 0<v \leq 1 \\
v^{1 / 2} & \leq C F(v), & & 1 \leq v .
\end{aligned}
$$


Observe that then, formally,

$$
\begin{aligned}
|F(v(x, t))| & \leq \int \mid F\left(v(x, t)_{x} \mid d x\right. \\
& =\int \psi^{\prime}(v)^{1 / 2} \varepsilon(v)\left|v_{x}\right| d x \leq\left(\int \psi(v) d x\right)^{1 / 2}\left(\int E_{x}^{2} d x\right)^{1 / 2} .
\end{aligned}
$$

A pointwise bound for $F(v(x, t))$ would then follow from time independent bounds for the two integrals on the right. Together with (3.5), this would then prove (3.4). Actually, we shall develop the entire argument at the discrete level, making suitable modifications to take account of the jump in $v$.

We therefore analyze the solution $\left(v_{j}(t), u_{k}(t)\right)$ of the difference scheme (2.1)(2.3) assuming that

$$
\begin{gathered}
0<1 / K_{0} \leq v_{j}(0) \leq K_{0} \\
\sum_{k} u_{k}(0)^{2} h+\sum_{j} \psi\left(v_{j}(0)\right) h \leq K_{0}
\end{gathered}
$$

and

$$
\sum_{k \neq 0} \delta v_{k}(0)^{2} h+\left|[v]_{0}(0)\right| \leq K_{0}
$$

As before, we let

$$
[E]_{0}(t)=E_{1 / 2}(t)-E_{-1 / 2}(t)
$$

Now fix $T$ and define $\underline{v}(T)$ and $\bar{v}(T)$ by

$$
\underline{v}=\inf _{\substack{0 \leq t \leq T \\ j}} v_{j}(t) \quad \text { and } \quad \bar{v}=\sup _{\substack{0 \leq t \leq T \\ j}} v_{j}(t) .
$$

Then $\infty>\bar{v} \geq \underline{v}>0$ by Theorem 2.1. Our plan is to estimate sums corresponding to the integrals on the right-hand side of the energy estimate (2.12) in terms of $\underline{v}$ and $\bar{v}$, and then to use a discrete version of the estimate (3.6) to bound $F$. The argument will close, via (3.5), when $\gamma>3 / 2$.

Now, (2.4) shows that

$$
\left|u_{0}\right| \leq\left|u_{1}\right|+\left|u_{-1}\right|+C h \underline{v}^{-\gamma} \bar{v}
$$

since, by (2.13) and (2.14),

$$
\left|[p]_{0}(t)\right|=\left|\alpha(t)[E]_{0}(t)\right| \leq C \underline{v}^{-\gamma}\left|[E]_{0}(0)\right| .
$$

Consequently,

$$
\sum_{k} u_{k}^{2} h \leq C \sum_{k \neq 0} u_{k}^{2} h+C h^{3} \underline{v}^{-2 \gamma} \bar{v}^{2} \leq C\left(1+h^{3} \underline{v}^{-2 \gamma} \bar{v}^{2}\right)
$$

by (2.11). A simple discrete Sobolev estimate then shows that

$$
\begin{aligned}
u_{0}^{2} & \leq C\left(\sum_{k} u_{k}^{2} h\right)^{1 / 2}\left(\sum_{j} \delta u_{j}^{2} h\right)^{1 / 2} \\
& \leq C\left(1+h^{3 / 2} \underline{v}^{-\gamma} \bar{v}\right) \bar{v}^{1 / 2}\left(\sum_{j} \varepsilon_{j} \delta u_{j}^{2} h\right)^{1 / 2} .
\end{aligned}
$$


Thus

$$
\left|u_{0}\right| \leq C \bar{v}^{1 / 4}\left(1+h^{3 / 4} \underline{v}^{-\gamma / 2} \bar{v}^{1 / 2}\right)\left(\sum_{j} \varepsilon_{j} \delta u_{j}^{2} h\right)^{1 / 4} .
$$

We can now estimate the integral on the right side of (2.12). Using (2.11) and (2.13), we have

$$
\begin{aligned}
\left|\int_{0}^{t} u_{0}(s)[\dot{E}]_{0}(s) d s\right| \leq & C \bar{v}^{1 / 4}\left(1+h^{3 / 4} \underline{v}^{-\gamma / 2} \bar{v}^{1 / 2}\right) \\
& \cdot \int_{0}^{t}\left(\sum_{j} \varepsilon_{j}(s) \delta u_{j}(s)^{2} h\right)^{1 / 4}|\alpha(s)| \exp \left[\int_{0}^{s} \alpha(\tau) d \tau\right] d s \\
\leq & C \bar{v}^{1 / 4}\left(1+h^{3 / 4} \underline{v}^{-\gamma / 2} \bar{v}^{1 / 2}\right)\left(\int_{0}^{t} \sum_{j} \varepsilon_{j} \delta u_{j}^{2} h d s\right)^{1 / 4} \\
& \cdot\left(\int_{0}^{t}|\alpha(s)|^{4 / 3} \exp \left[\frac{4}{3} \int_{0}^{s} \alpha(\tau) d \tau\right] d s\right)^{3 / 4} \\
\leq & C \bar{v}^{1 / 4}\left(1+h^{3 / 4} \underline{v}^{-\gamma / 2} \bar{v}^{1 / 2}\right) \cdot C \\
& \cdot \sup _{s}|\alpha(s)|^{1 / 4}\left|\int_{0}^{t} \frac{d}{d s} \exp ^{-\gamma}\left[\frac{4}{3} \int_{0}^{s} \alpha(\tau) d \tau\right] d s\right|^{3 / 4} \\
\leq & C \underline{v}^{-\gamma / 4} \bar{v}^{1 / 4}\left(1+h^{3 / 4} \underline{v}^{-\gamma / 2} \bar{v}^{1 / 2}\right) .
\end{aligned}
$$

Without loss of generality, we may take $C \underline{v}^{-\gamma / 4} \bar{v}^{1 / 4} \geq 1$. Then from the above estimate and (2.12) we obtain

$$
\sum_{k \neq 0} \delta E_{k}(t)^{2} h \leq C \underline{v}^{-\gamma / 4} \bar{v}^{1 / 4}\left(1+h^{3 / 4} \underline{v}^{-\gamma / 2} \bar{v}^{1 / 2}\right), \quad 0 \leq t \leq T .
$$

We now use this bound, together with $(2.11)$ of Lemma 2.1 , to bound $F\left(v_{j}(t)\right)$. Fix $j$ and, without loss of generality, take $j<0$. Then since

$$
\frac{d F}{d E}=\frac{d F}{d v} / \frac{d E}{d v}=\psi^{1 / 2},
$$

we obtain

$$
\begin{aligned}
\left|F\left(v_{j}(t)\right)\right| & \leq \sum_{k<0}\left|\delta F_{k}(t)\right| h=\sum_{k<0} \psi\left(\tilde{v}_{k}\right)^{1 / 2}\left|\delta E_{k}\right| h \\
& \leq C\left(\sum_{j<0} \psi_{j} h\right)^{1 / 2}\left(\sum_{k<0} \delta E_{k}^{2} h\right)^{1 / 2} \\
& \leq C \underline{v}^{-\gamma / 8} \bar{v}^{1 / 8}\left(1+h^{3 / 8} \underline{v}^{-\gamma / 4} \bar{v}^{1 / 4}\right) .
\end{aligned}
$$

(The same bound holds for $j>0$.) Now, (3.5) shows that

$$
v^{-\gamma / 8} \leq C F(v)^{\gamma /(4 \gamma-4)}, \quad v \leq v^{\prime}
$$

and

$$
v^{1 / 8} \leq C F(v)^{1 / 4}, \quad v \geq v^{\prime}
$$


so that, if $D(T) \equiv \underline{v}^{\gamma / 8} \bar{v}^{1 / 8}$, then by $(3.8)$,

$$
\begin{aligned}
D & \leq C \sup F\left(v_{j}(t)\right)^{(2 \gamma-1) /(4 \gamma-4)} \\
& \leq C\left[D\left(1+h^{3 / 8} D^{2}\right)\right]^{(2 \gamma-1) /(4 \gamma-4)}
\end{aligned}
$$

Thus

$$
D(T)^{(2 \gamma-3) /(4 \gamma-4)} \leq C\left(1+h^{3 / 8} D(T)^{2}\right)^{(2 \gamma-1) /(4 \gamma-4)} .
$$

Fix the constant $C$ in (3.9) and define

$$
\bar{D} \equiv \max \left[2 D(0),(2 C)^{(4 \gamma-4) /(2 \gamma-3)}\right] .
$$

We claim that, if $h$ is sufficiently small, depending on $\bar{D}$, then $D(T)<\bar{D}$ for all $T$. This is certainly true, for given $h$, if $T$ is small. If for some $T, D(T)=\bar{D}$, then (3.9) implies that

$$
D(T)^{(2 \gamma-3) /(4 \gamma-4)} \leq C\left[1+h^{3 / 8} \bar{D}^{2}\right]^{(2 \gamma-1) /(4 \gamma-4)} \leq \frac{3}{2} C
$$

for $h$ small, so that

$$
D(T) \leq\left(\frac{3}{2} C\right)^{(4 \gamma-4) /(2 \gamma-3)}
$$

a contradiction.

We have thus shown that $\underline{v}(T)^{-\gamma / 8} \bar{v}(T)^{1 / 8} \leq \bar{D}$ for all $T \geq 0$. Since $\bar{v} \geq v^{\prime} \geq \underline{v}$, (3.4) is proved. Theorem 3.1 then follows easily upon passing to the limit as $h \rightarrow 0$. We omit the details.

\section{REFERENCES}

1. David Hoff, Construction of solutions for compressible, isentropic Navier-Stokes equations in one space dimension with nonsmooth initial data, Proc. Roy. Soc. Edinburgh Sect. A 103 (1986), 301-315.

2. David Hoff and Tai-Ping Liu, (to appear).

3. David Hoff and Joel Smoller, Solutions in the large for certain nonlinear parabolic systems, Ann. Inst. Henri Poincaré, Analyse Non linéaire 2 (1985), 213-235.

4. A. Kazhikov and V. Shelukhin, Unique global solutions in time of initial boundary value problems for one dimensional equations of a viscous gas, Prikl. Mat. Meh. 41 (1977), 282-291= J. Appl. Math. Mech. 41 (1977), 273-283.

6. Jong Uhn Kim, Global existence of solutions of the equations of one dimensional thermoviscoelasticity with initial data in $B V$ and $L^{1}$, Ann. Scuola Norm. Sup. Pisa 10 (1983), 357-427.

7. Denis Serre, Solutions faibles globales des équations de Navier-Stokes pour un fluide compressible, preprint.

Department of Mathematics, Indiana University, Bloomington, Indiana 